DESY M-84-15

May 1984

\title{
Theoretical Prediction \\ of Head Tail Tune Shift \\ in PETRA
}

by

R. Klatt, R.D. Kohaupt, T. Weiland 
DESY behält sich alle Rechte für den Fall der Schutzrechtserteilung und für die wirtschaftliche Verwertung der in diesem Bericht enthaltenen Informationen vor.

DESY reserves all rights for commercial use of information included in this report, especially in case of filing application for or grant of patents.

"Die Verantwortung für den Inhatt dieses Internen Berichtes liegt ausschiießlich beim Verfasser" 


\section{Abstract}

The shift in frequency of the fundamental $m=0$ head tail mode in a storage ring can be calculated from the optics and wake potentials. Such calculations have been performed for various beam pipe components in PETRA and are summarized here. Comparision with measurements shows good agreement for long bunches and that the theoretical description of the impedance is incomplete for short bunches (high frequencies).

\section{List of symbols}

q charge

$w_{\delta \underline{1}}(s)$ wake potential of a point charge at distance $s$ behind it $\Psi_{\lambda \perp}(s)$ wake potential inside a bunch of longitudinal line charge density $\lambda(s)$

$\lambda(s)$ line charge density

B beta function at the location of the impedance

$k_{1} \quad$ transverse kick parameter

$E_{0} \quad$ nominal particle energy

$\Delta p_{\perp} \quad$ transverse momentum

c speed of light

$f_{z} \quad$ vertical betatron frequency

$f_{x} \quad$ horizontal betatron frequency 


\section{Introduction}

The shift in oscillation frequency of the head tail bunch modes in a storage ring results from wake field forces excited in many objects such as rf cavities, separator tanks and vacuum chamber joints. The wake field force, integrated over the transit time, is a wake potential that describes the distribution of momentum change inside a bunch of particles. If the beam offset from the ideal orbit is small the transverse wake potential gives the transverse kick imported by one particle to a second one at distance s:

$$
\begin{array}{ll}
c \cdot \Delta p_{\perp}^{l \rightarrow 2}=q_{1} \cdot r_{1} w_{\delta \perp}(s) & s>0 \\
c \cdot \Delta p_{\perp}^{l \rightarrow 2}=0 & s<0
\end{array}
$$

The kick experienced by test particle 非 2 does not depend on its own radial position (i.e. offset). Taking $r_{l}$ as the bunch offsett and $q_{l}$ as the total charge in a bunch we obtain a kick distribution inside the bunch given by:

$$
c \cdot \Delta p_{\perp}(s)=q_{1} \cdot r_{1} \cdot w_{\lambda \perp}(s)
$$

A typical result is shown in figure 1.

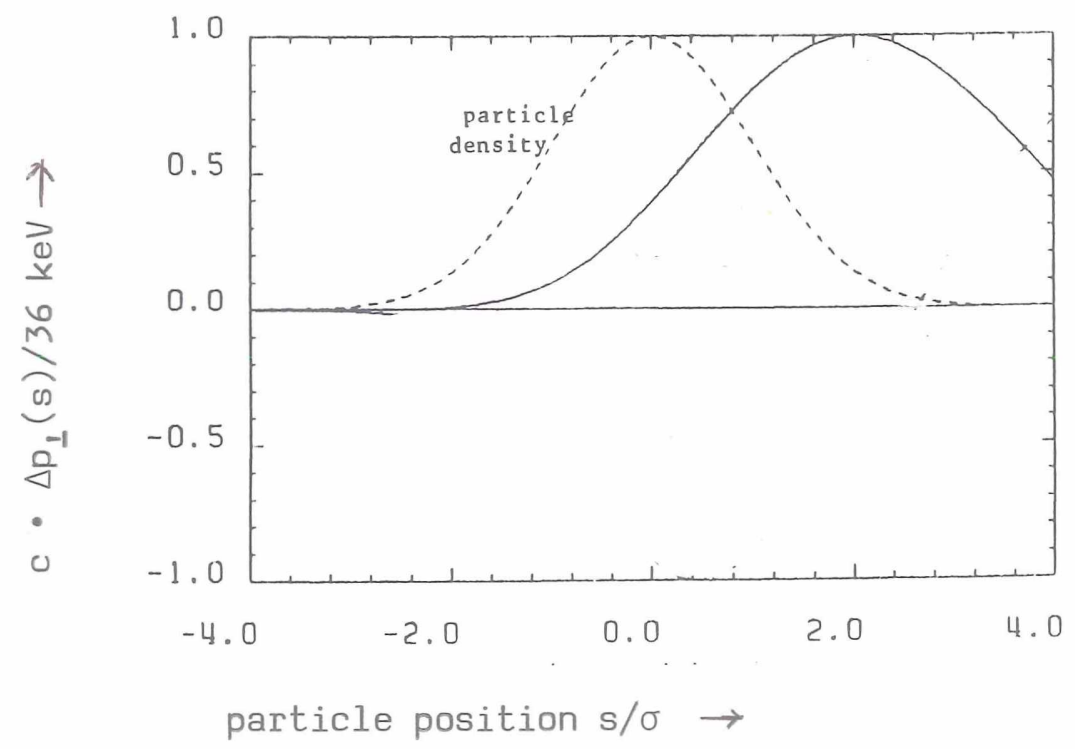

Figure 1: Transverse kick inside a Gaussian bunch after having passed a single cell PETRA accelerating cavity off axis (total charge $=1 \mu \mathrm{C}$, beam offset $=0.5 \mathrm{~cm}, \sigma=2 \mathrm{~cm}$ ) 
The dipole moment of the total kick per unit offset $\mathrm{r}_{1}$ and per unit charge $\mathrm{q}_{l}$ inside a bunch with longitudinal density $\lambda(\mathrm{s})$ can be calculated as ${ }^{*}$ )

$$
k_{\perp}(\lambda)=\frac{\int_{-\infty 0}^{+\infty o} \lambda(x) w_{\lambda \perp}(x) d x}{\left[\int_{-\infty}^{+\infty} \lambda(x) d x\right]^{2}}
$$

This "transverse kick parameter" has its equivalent in the case of higher order mode losses where one uses the loss parameter $k$.

The betatron tune shift of the $\mathrm{m}=0$ mode due to a quadrupole error is given by ${ }^{1}$ :

$$
\Delta Q=\frac{-1}{4 \pi} \underset{\substack{\text { circum- } \\ \text { ference }}}{\infty \mathrm{s}(\mathrm{s}) B(\mathrm{~s}) \mathrm{ds}}
$$

Since the wake force behaves like a quadrupole $(\vec{F} \propto \vec{r})$ we can obtain the tune shift from the wake potential as ${ }^{2}$ :

$$
\begin{aligned}
\Delta Q & =\frac{-\mathrm{e}}{4 \pi E_{0}}\langle\beta\rangle \underset{\text { bunch }}{\int} \lambda(x) w_{\lambda}(x) d x \\
& =\frac{-e}{4 \pi E_{0}}\langle\beta\rangle \cdot k_{1} \cdot q
\end{aligned}
$$

For all objects of cylindrical symmetry we can use TBCI $^{2}$ to obtain correct values $^{3}$ for the transverse kick parameters.

For nonsymmetric objects we need a fully threedimensional BCI program. Since this program is not yet in its final shape and not yet sufficiently tested we use cylindrical models of 3D objects.

Figure $2 a$ shows the layout of all the cavity types in PETRA and figure 2b shows the cross section of PETRA vacuum chamber joint. The vacuum chamber joints are modeled by circular ones having the chamber height as radius. Figure $2 \mathrm{~b}$ also shows the quadrupole chamber joints and separator tanks in PETRA.

In table I, we list the transverse kick parameter $k_{\perp}$ for each of these components at various bunch lengths (assuming Gaussian shape).

*) $k_{\perp}$ is directly calculated by the TBCI program 

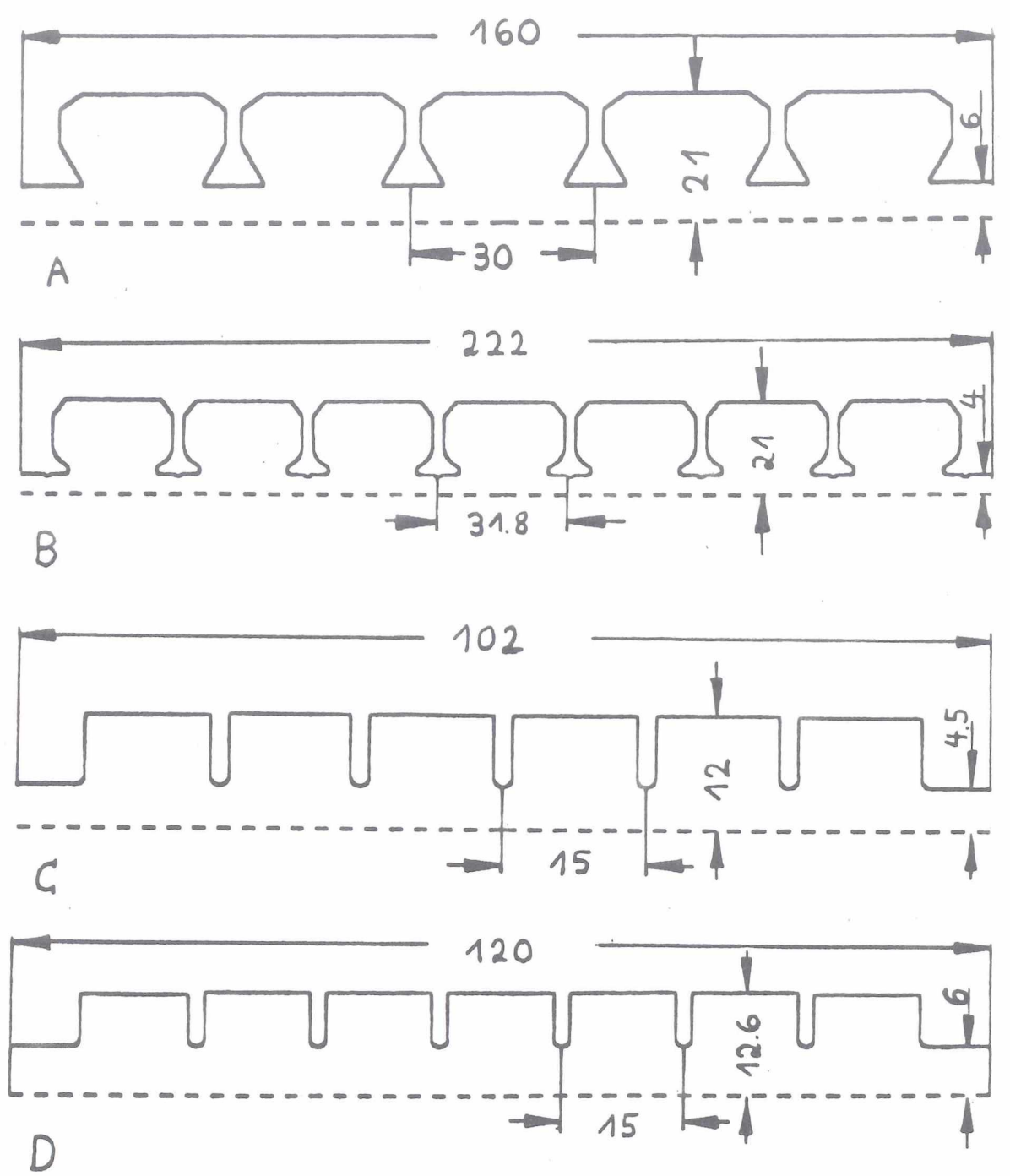

Figure 2a: Layout of PETRA cavities
A $500 \mathrm{MHz}$ five cell
B $500 \mathrm{MHz}$ seven cell
C $1 \mathrm{GHz}$ six cell
D $1 \mathrm{GHz}$ seven cell 

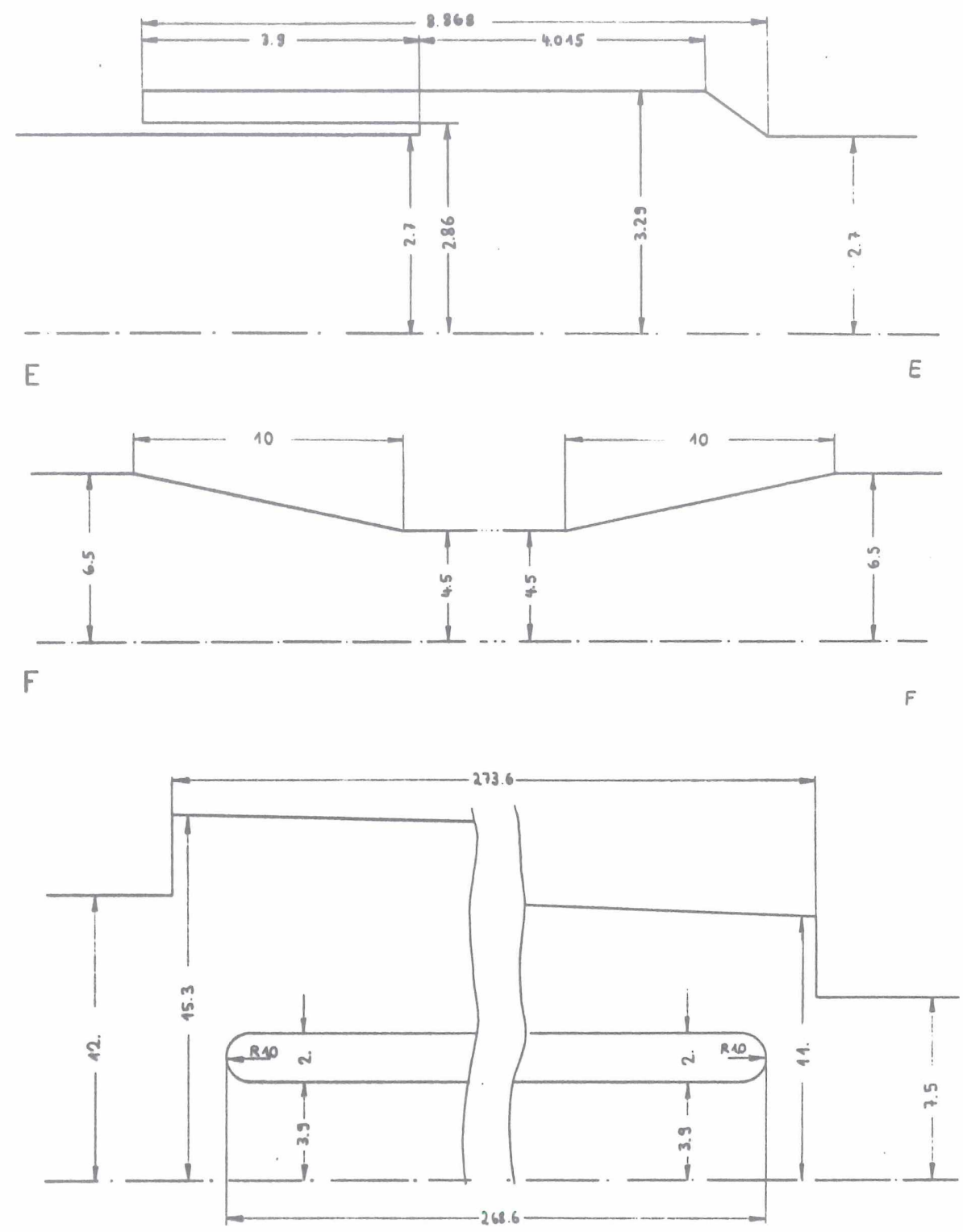

G

Figure 2b: Geometry of various components in PETRA

$E$ vacuum chamber joint, bending magnet

$F$ vacuum chamber joint, quadrupole magnet

G separator tank 
In order to simplify the use of table I we give the formula for the tune shift in PETRA as:

$$
\frac{\Delta f}{H z}=-\frac{0.01135}{\left(E_{0} / 7 \mathrm{GeV}\right)}\left(\frac{I_{b}}{m A}\right)\left(\frac{<\beta>}{m}\right)\left(\frac{k_{\perp}}{V / p C m}\right)
$$

Table I: Transverse kick parameter of various components in PETRA

A: 5 cell $500 \mathrm{MHz}$ cavity

\begin{tabular}{|l|lllll|}
\hline$\sigma / \mathrm{cm}$ & 1 & 2 & 3 & 4 & 5 \\
$\mathrm{k}_{\mathfrak{1}} /(\mathrm{V} / \mathrm{pCm})$ & 10 & 14 & 15 & 15 & 13 \\
\hline
\end{tabular}

B: 7 cell $500 \mathrm{MHz}$ cavity

\begin{tabular}{|l|lllll|}
\hline$\sigma / \mathrm{cm}$ & 1 & 2 & 3 & 4 & 5 \\
$\mathrm{k}_{1} /(\mathrm{V} / \mathrm{pCm})$ & 32 & 39 & 37 & 33 & 29 \\
\hline
\end{tabular}

C: 6 cell $1000 \mathrm{MHz}$ cavity

\begin{tabular}{|l|lllll|}
\hline$\sigma / \mathrm{cm}$ & 1 & 2 & 3 & 4 & 5 \\
$\mathrm{k}_{\perp} /(\mathrm{V} / \mathrm{pCm})$ & 19 & 21 & 20 & 17 & 14 \\
\hline
\end{tabular}

D: 7 cell $1000 \mathrm{MHz}$ cavity

\begin{tabular}{|l|lllll|}
\hline$\sigma / \mathrm{cm}$ & 1 & 2 & 3 & 4 & 5 \\
$\mathrm{k}_{\perp} /(\mathrm{V} / \mathrm{pCm})$ & 10 & 12 & 12 & 11 & 9 \\
\hline
\end{tabular}

E: Vacuum chamber joint in arc (vertical)

\begin{tabular}{|l|lllll|}
\hline$\sigma / \mathrm{cm}$ & 1 & 2 & 3 & 4 & 5 \\
$\mathrm{k}_{\perp} /(\mathrm{V} / \mathrm{pCm})$ & 3.7 & 2.8 & 2.0 & 1.5 & 1.1 \\
\hline
\end{tabular}

F: Vacuum chamber joint in straight section (vertical and horizontal)

\begin{tabular}{|l|lllll|}
\hline$\sigma / \mathrm{cm}$ & 1 & 2 & 3 & 4 & 5 \\
$\mathrm{k}_{\underline{1}} /(\mathrm{V} / \mathrm{pCm})$ & 0.86 & 0.73 & 0.52 & 0.36 & 0.26 \\
\hline
\end{tabular}

G: Separator tank (vertical)

\begin{tabular}{|l|lllll|}
\hline$\sigma / \mathrm{cm}$ & 1 & 2 & 3 & 4 & 5 \\
$\mathrm{k}_{1} /(\mathrm{V} / \mathrm{pCm})$ & 15 & 22 & 25 & 27 & 27 \\
\hline
\end{tabular}




\section{Examples}

For PETRA at injection energy with a MIl00 optics and

- 60 five cell cavities

- 232 vacuum chamber joints type E

- 48 vacuum chamber joints type F

- 8 separator tanks

we obtain

\begin{tabular}{|c|l|l|l|l|l|}
\hline$\sigma / \mathrm{cm}$ & 1 & 2 & 3 & 4 & 5 \\
\hline$\Delta \mathrm{f}_{\mathrm{z}} / \mathrm{Hz}$ & 276 & 290 & 271 & 252 & 215 \\
$\Delta \mathrm{f}_{\mathrm{x}} / \mathrm{Hz}$ & 109 & 149 & 157 & 156 & 135 \\
\hline
\end{tabular}

However measurements ${ }^{4}$ show much higher vertical tune shift than predicted, namely:

\begin{tabular}{|l|c|c|}
\hline$\sigma / c m$ & $\Delta f_{z} / H z$ theory & $\Delta f_{z} / H z$ measured \\
\hline 1.05 & 277 & 580 \\
2.10 & 273 & 445 \\
5.00 & 215 & 270 \\
\hline
\end{tabular}

More recently, using, for example, the optics MIIO0G and with

- 56 five cell cavities $500 \mathrm{MHz}$

- 56 seven cell cavities $500 \mathrm{MHz}$

- 16 six cell cavities $1000 \mathrm{MHz}$

- 8 seven cell cavities $1000 \mathrm{MHz}$

- 288 vacuum chamber joints type $E$

- 56 vacuum chamber joints type F

- 8 separator tanks

we obtain

\begin{tabular}{|l|l|l|l|l|l|}
\hline$\sigma / \mathrm{cm}$ & $\mathrm{l}$ & 2 & 3 & 4 & 5 \\
\hline$\Delta \mathrm{f}_{\mathrm{z}} / \mathrm{Hz}$ & 676 & 753 & 704 & 633 & 544 \\
$\Delta \mathrm{f}_{\mathrm{x}} / \mathrm{Hz}$ & 474 & 586 & 572 & 522 & 453 \\
\hline
\end{tabular}


In this case measurements show

\begin{tabular}{|l|c|c|}
\hline$\sigma / \mathrm{cm}$ & $\Delta \mathrm{f}_{\mathrm{z}} / \mathrm{Hz}$ theory & $\Delta \mathrm{f}_{\mathrm{z}} / \mathrm{Hz}$ measured \\
\hline 1.04 & 679 & 960 \\
4.85 & 463 & 490 \\
\hline
\end{tabular}

\section{Conclusions}

We find a clear discrepancy between measured and predicted tune shifts especially for short bunches $(\sigma=1 \mathrm{~cm})$. For long bunches however, we find good agreement and also no significant discrepancy between the tune shift in both planes, horizontal or vertical.

It follows that for long bunches the symmetric impedance is dominated by the cavities and that predictions are quite accurate.

For short bunches the impedance is dominated by vacuum chamber joints and other unindentified components outside the cavity region.

In order to complete our theoretical knowledge of this complex system we must do more accurate computations with the threedimensional BCI program ${ }^{5}$ and investigate other sources of non-geometric impedance such as aluminium oxide in the vacuum system ${ }^{6}$.

\section{Literature}

/I/ E.D. Courant, H.S. Snyder, Annuals of Physics 3, 1958

/2/ T. Weiland, DESY 82-015, March 1982

/3/ K. Bane, T. Weiland, Proceedings of the 12th International Conference on High Energy Accelerators, Fermilab 1983, P. 317-319 and SLAC, AP-1, 1983

14/ R.D. Kohaupt, T. Weiland, DESY M 82-07 and recent measurements performed by the members of the PETRA group

15/ T. Weiland, Three dimensional BCI programm, no yet published

/6/ A. Piwinski, private communication

5. Acknowledgements

The authors wish to thank D.P. Barber for careful reading the manuscript. 